\title{
Fluorine F 18 Fluorobenzyl Triphenyl Phosphonium
}

National Cancer Institute

\section{Source}

National Cancer Institute. Fluorine F 18 Fluorobenzyl Triphenyl Phosphonium. NCI

Thesaurus. Code C117294.

A radioconjug ate and cationic lipophilic agent consisting of fluorobenzyl triphenyl phosphonium (FBnTP), labeled with the radioisotope fluorine $F 18$, with potential use as a tracer for both mitochondrial membrane potential (MMP) and apoptosis, and as a tumor imaging agent using positron emission tomography (PET). Upon administration, fluorine F 18 FBnTP is taken up by cells and its uptake and accumulation within mitochondria is directly correlated with MMP. Apoptosis causes a loss of membrane potential across the inner mitochondrial membrane which decreases FBnTP mitochondrial uptake. As apoptosis is suppressed in tumor cells, the FBnTP uptake is increased as compared to normal cells. This allows, upon PET, for the imaging of cancer cells. As apoptotic-inducing chemotherapeutic agents cause a collapse of MMP, this agent can also be used to assess the response of tumor cells to those chemotherapeutic agents. 